EUROPEAN JOURNAL OF PURE AND APPLIED MATHEMATICS

Vol. 13, No. 1, 2020, 170-179

ISSN 1307-5543 - www.ejpam.com

Published by New York Business Global

\title{
On Strong Resolving Domination in the Join and Corona of Graphs
}

\author{
Gerald B. Monsanto ${ }^{1, *}$, Penelyn L. Acal ${ }^{2}$, Helen M. Rara ${ }^{3}$ \\ 1 Department of Teacher Education, Visayas State University-Villaba, 6537 Villaba, Leyte, \\ Philippines \\ 2 Department of Mathematical Sciences, University of Science and Technology of Southern \\ Philippines, 9023, Cagayan de Oro City, Philippines \\ 3 Department of Mathematics and Statistics, College of Science and Mathematics, Center \\ of Graph Theory, Algebra, and Analysis-Premier Research Institute of Science and \\ Mathematics, Mindanao State University-Iligan Institute of Technology, 9200 Iligan City, \\ Philippines
}

\begin{abstract}
Let $G$ be a connected graph. A subset $S \subseteq V(G)$ is a strong resolving dominating set of $G$ if $S$ is a dominating set and for every pair of vertices $u, v \in V(G)$, there exists a vertex $w \in S$ such that $u \in I_{G}[v, w]$ or $v \in I_{G}[u, w]$. The smallest cardinality of a strong resolving dominating set of $G$ is called the strong resolving domination number of $G$. In this paper, we characterize the strong resolving dominating sets in the join and corona of graphs and determine the bounds or exact values of the strong resolving domination number of these graphs.
\end{abstract}

2020 Mathematics Subject Classifications: 05C69

Key Words and Phrases: Strong resolving dominating set, strong resolving domination number, join, corona

\section{Introduction}

All graphs considered in this study are finite, simple, and undirected connected graphs, that is, without loops and multiple edges. For some basic concepts in Graph Theory, we refer readers to [4].

Let $G=(V(G), E(G))$ be a connected graph. The open neighborhood $N_{G}(v)=\{u \in$ $V(G): u v \in E(G)\}$. Any element $u$ of $N_{G}(v)$ is called a neighbor of $v$. The closed neighborhood $N_{G}[v]=N_{G}(v) \cup\{v\}$. Thus, the degree of $v$ is given by $\operatorname{deg}_{G}(v)=\left|N_{G}(v)\right|$. Customarily, for $S \subseteq V(G), N_{G}(S)=\bigcup_{v \in S} N_{G}(v)$ and $N_{G}[S]=\bigcup_{v \in S} N_{G}[v]$.

*Corresponding author.

DOI: https://doi.org/10.29020/nybg.ejpam.v13i1.3625

Email addresses: monger2006@yahoo.com (G. Monsanto),

penelyn.acal@g.msuiit.edu.ph (P. Acal), helen.rara@g.msuiit.edu.ph (H. Rara) 
A nonempty set $S \subseteq V(G)$ is a dominating set in graph $G$ if $N_{G}[S]=V(G)$. Otherwise, we say $S$ is a non-dominating set of $G$. The domination number of a graph $G$, denoted by $\gamma(G)$, is given by $\gamma(G)=\min \{|S|: S$ is a dominating set of $G\}$. If $|S|=\gamma(G)$, then $S$ is said to be a minimum dominating set or $\gamma$-set of $G$.

A vertex $w \in S$ strongly resolves two different vertices $u, v \in V(G)$ if $v \in I_{G}[u, w]$ or if $u \in I_{G}[v, w]$. A set $W$ of vertices in $G$ is a strong resolving set of $G$ if every two vertices of $G$ are strongly resolved by some vertex of $W$. The smallest cardinality of a strong resolving set of $G$ is called the strong metric dimension of $G$ and is denoted by $\operatorname{sdim}(G)$.

A subset $S \subseteq V(G)$ is a strong resolving dominating set of $G$ if it is both strong resolving and dominating. The smallest cardinality of a strong resolving dominating set of $G$ is called the strong resolving domination number of $G$ and is denoted by $\gamma_{s r}(G)$. A strong resolving dominating set of cardinality $\gamma_{s r}(G)$ is called a $\gamma_{s r}$-set of $G$.

A clique in a graph $G$ is a complete induced subgraph of $G$. A clique $C$ in $G$ is called a superclique if for every pair of distinct vertices $u, v \in C$, there exists $w \in V(G) \backslash C$ such that $w \in N_{G}(u) \backslash N_{G}(v)$ or $w \in N_{G}(v) \backslash N_{G}(u)$. A superclique $C$ in $G$ is called a dominated superclique if for every $u \in C$, there exists $v \in V(G) \backslash C$ such that $u v \in E(G)$ [3]. A superclique (resp. dominated superclique) $C$ is maximum in $G$ if $|C| \geq\left|C^{*}\right|$ for all supercliques (resp. dominated supercliques) $C^{*}$ in $G$. The superclique (resp. dominated superclique) number, $\omega_{S}(G)$ (resp. $\omega_{D S}(G)$ ) of $G$ is the cardinality of a maximum superclique (resp. maximum dominated superclique) in $G$.

In recent years, the concept of domination in graphs has been studied extensively and several research papers have been published on this topic. The said concept was not formally defined mathematically until the publications of the books by Claude Berge [1] in 1958 and Oystein Ore in 1962. In 1977, a survey paper by Cockayne and Hedetniemi [2] began to study the concept of domination.

On the other hand, the problem of uniquely recognizing the possible position of an intruder such as fault in a computer network and spoiled device was the principal motivation in introducing the concept of metric dimension in graphs.

Slater [6] brought in the notion of locating sets and its minimal cardinality as locating number. The same concept was also introduced by Harary and Melter [4] but using the terms resolving sets and metric dimension to refer to locating sets and locating number, respectively.

In 2007, Oellerman and Peter-Fransen [5] introduced the strong resolving graph $G_{S R}$ of a connected graph $G$ as a tool to study the strong metric dimension of $G$.

This study aims to define and characterize the strong resolving dominating sets and determine the exact values or bounds in the join and corona of two graphs.

\section{Preliminary Results}

Remark 1. Every strong resolving dominating set of a connected graph $G$ is a dominating set. Hence, $\gamma(G) \leq \gamma_{s r}(G)$.

Remark 2. Every strong resolving dominating set of a connected graph $G$ is a strong resolving set. Thus, $\operatorname{sdim}(G) \leq \gamma_{s r}(G)$. 
Remark 3. For any connected graph $G$ of order $n, 1 \leq \gamma_{s r}(G) \leq n-1$.

Remark 4. Any superset of a strong resolving dominating set is a strong resolving dominating set.

Proposition 1. Let $G$ be a connected graph of order $n \geq 2$. Then,

(i) $\gamma_{s r}\left(P_{n}\right)=\left\lceil\frac{n+1}{3}\right\rceil$

(ii) $\gamma_{s r}\left(K_{n}\right)=n-1$

(iii)

$$
\gamma_{s r}\left(C_{n}\right)=\left\{\begin{array}{lll}
2 & , & \text { if } n=3 \\
n-2 & , & \text { if } n>3 \text { and } n \text { is odd } \\
\left\lceil\frac{n}{2}\right\rceil & , & \text { if } n>3 \text { and } n \text { is even }
\end{array}\right.
$$

Proposition 2. Let $G$ be a connected graph of order $n$ and let

$$
A=\left\{x \in V(G): \operatorname{deg}_{G}(x)=n-1\right\} .
$$

If $A \neq \varnothing$ and $C$ is a superclique in $G$, then $|C \cap A| \leq 1$. Moreover, if $C$ is a maximum superclique of $G$, then $|C \cap A|=1$.

Remark 5. Let $G$ be a nontrivial connected graph with $\operatorname{diam}(G) \leq 2$. For distinct vertices $u, v, w \in G, u \in I_{G}[v, w]$ if and only if $d_{G}(v, w)=2$ and $u \in N_{G}(v) \cap N_{G}(w)$.

Proposition 3. Let $G$ be a nontrivial connected graph with $\operatorname{diam}(G) \leq 2$. Then $S=$ $V(G) \backslash C$ is a strong resolving set of $G$ if and only if $C=\varnothing$ or $C$ is a superclique in $G$. In particular, $\operatorname{sdim}(G)=|V(G)|-\omega_{S}(G)$.

Proof: Assume that $S$ is a strong resolving set of $G$. If $S \cap V(G)=V(G)$, then $C=\varnothing$. Suppose $S \subsetneq V(G)$. Let $C=V(G) \backslash S$. Then $S=V(G) \backslash C$. Let $x, y \in C$, where $x \neq y$. Since $S$ is a strong resolving set of $G, x$ and $y$ are strongly resolved by some $z \in S$. We may assume that $x \in I_{G}[y, z]$. Then $d_{G}(y, z)=2$ and $x \in N_{G}(y) \cap N_{G}(z)$ by Remark 5 . Thus, $z \in N_{G}(x) \backslash N_{G}(y)$, showing that $C$ is a superclique in $G$.

Conversely, assume that $S=V(G) \backslash C$, where $C$ is a superclique in $G$. Let $x, y \notin S$, where $x \neq y$. Then $x, y \in C$. Since $C$ is a superclique in $G$, there exists $z \in S$ such that $z \in N_{G}(x) \backslash N_{G}(y)$ or $z \in N_{G}(y) \backslash N_{G}(x)$. Since $\operatorname{diam}(G)=2, d_{G}(y, z)=2$ or $d_{G}(x, z)=2$. By Remark $5, x \in I_{G}[y, z]$ or $y \in I_{G}[x, z]$. Hence, $S$ is a strong resolving set of $G$.

Suppose $S$ is a strong resolving set of $G$. Then $S=V(G) \backslash C$, where $C$ is a superclique in $G$ and $|C|=\omega_{S}(G)$. Thus, $\operatorname{sdim}(G)=|S|=|V(G)|-|C|=|V(G)|-\omega_{S}(G)$.

\section{On Strong Resolving Domination in the Join of Graphs}

The join of two graphs $G$ and $H$ is the graph $G+H$ with vertex set $V(G+H)=$ $V(G) \dot{\cup} V(H)$ and edge set $E(G+H)=E(G) \dot{\cup} E(H) \cup\{u v: u \in V(G), v \in V(H)\}$. 
Remark 6. For the joins $\langle v\rangle+P_{n}$ and $\langle w\rangle+C_{n}$, it can be verified that $\gamma_{s r}\left(\langle v\rangle+P_{n}\right)=n-1$ for $n \geq 3$ and $\gamma_{s r}\left(\langle w\rangle+C_{n}\right)=n-2$ for $n \geq 4$.

Proposition 4. Let $G$ be a connected graph with $\gamma(G) \neq 1$ and let $K_{1}=\langle v\rangle$. Then $C \subseteq V\left(K_{1}+G\right)$ is a superclique of $K_{1}+G$ if and only if $|C|=1$ or $|C| \geq 2$ and $C \backslash\{v\}$ is a superclique of $G$.

Proof: The conditions follow immediately if $C \subseteq V\left(K_{1}+G\right)$ is a superclique of $K_{1}+G$. For the converse, the case when $|C|=1$ is obvious. Suppose $|C| \geq 2$. Since $C \backslash\{v\}$ is a superclique of $G$, we only need to consider the pair of distinct vertices $z, v \in C$. Since $\gamma(G) \neq 1$, there exists $w \in V(G)$ such that $z w \notin E(G)$. Since $\operatorname{diam}\left(K_{1}+G\right)=$ $2, d_{G}(z, w)=2$. Hence, $z \in N_{G}(v) \backslash N_{G}(w)$, showing that $C$ is a superclique of $K_{1}+G$.

Theorem 1. Let $G$ be a nontrivial connected graph of order $n$ with $\gamma(G) \neq 1$ and $K_{1}=\langle v\rangle$. Then $S \subseteq V\left(K_{1}+G\right)$ is a strong resolving dominating set of $K_{1}+G$ if and only if $S=V(G)$, or $S=V\left(K_{1}+G\right) \backslash C$ or $S=V(G) \backslash C^{*}$ where $C$ and $C^{*}$ are superclique and dominated superclique, respectively, in $G$.

Proof: Let $S$ be a strong resolving dominating set of $K_{1}+G$. Suppose $\gamma(G) \neq 1$. If $v \notin S$, then $S \subsetneq V(G)$. By Proposition 3, $S=V\left(K_{1}+G\right) \backslash(C \cup\{v\})=V(G) \backslash C$, where $S$ is a dominating set in $K_{1}+G$ and $C \cup\{v\}$ is a superclique in $K_{1}+G$. By Proposition 4, $C$ is a superclique in $G$. Since $\{v\}$ is a superclique in $K_{1}+G, S=V\left(K_{1}+G\right) \backslash\{v\}=V(G)$. On the other hand, if $v \in S$ and $C=V\left(K_{1}+G\right) \backslash S$, then $S=V\left(K_{1}+G\right) \backslash C$ where $C$ is a superclique in $K_{1}+G$ by Proposition 3. By Proposition 4, $C \backslash\{v\}=C$ is a superclique in $G$. Conversely, the case when $S=V(G)$ and $S=V\left(K_{1}+G\right) \backslash C$ follows immediately from Proposition 3. Suppose $S=V(G) \backslash C^{*}$, where $C^{*}$ is a dominated superclique in $G$. By Proposition 4, $C \cup\{v\}$ is a superclique of $K_{1}+G$. Since $v \notin S, S=V(G) \backslash C^{*}=$ $V\left(K_{1}+G\right) \backslash(C \cup\{v\})$. By Proposition 3, $S$ is a strong resolving dominating set of $K_{1}+G$.

Theorem 2. Let $G$ be a nontrivial connected graph of order $n$ with $\gamma(G)=1$ and $K_{1}=\langle v\rangle$. Then $S \subseteq V\left(K_{1}+G\right)$ is a strong resolving dominating set of $K_{1}+G$ if and only if $S=V(G)$ or $S=V\left(K_{1}+G\right) \backslash C$ or $S=\left(V(G) \backslash C^{*}\right) \cup\left\{x \in C^{*}: \operatorname{deg}(x)=n-1\right\}$ where $C$ and $C^{*}$ are superclique and dominated superclique, respectively, in $G$.

Proof: Let $S$ be a strong resolving dominating set of $K_{1}+G$. Suppose $\gamma(G)=1$. If $v \in S$ and $C=V\left(K_{1}+G\right) \backslash S$, then $S=V\left(K_{1}+G\right) \backslash C=\{v\} \cup(V(G) \backslash C)$. By Proposition $3, C$ is a superclique in $K_{1}+G$. Hence for $x, y \in C, x \neq y$, there exists $w \in V(G) \backslash C$ such that $w \in N_{G}(x) \backslash N_{G}(y)$ or $w \in N_{G}(y) \backslash N_{G}(x)$, showing that $C$ is a superclique in $G$. On the other hand, if $v \notin S$, then $S \subsetneq V(G)$. Let $C=V\left(K_{1}+G\right) \backslash S$. Hence, $S=V\left(K_{1}+G\right) \backslash C=V(G) \backslash C$. By Proposition 3, $C$ is a superclique in $K_{1}+G$. Hence, $C$ is also a superclique in $G$. Since $\gamma(G)=1, A_{G}=\left\{z \in V(G) ; \operatorname{deg}_{G}(z)=n-1\right\} \neq \varnothing$. By Proposition 2, $\left|C \cap A_{G}\right|=1$. Let $z \in C \cap A_{G}$. Since $d_{K_{1}+G}(z, v)=1$ and $d_{K_{1}+G}(v)=n$, none of the elements in $S$ strongly resolves $z$ and $v$, a contradiction. Hence, $z \in S$. Thus, $S=(V(G) \backslash C) \cup\{z\}$. In addition, since $\{v\}$ is a superclique in $K_{1}+G, S=$ 
$V\left(K_{1}+G\right) \backslash\{v\}=V(G)$. Similarly, if $C^{*}=V(G) \backslash S$, then $C^{*}$ is a dominated superclique in $G$.

For the converse, the case when $S=V(G)$ is trivial. Suppose $S=V\left(K_{1}+G\right) \backslash C$, where $C$ is a superclique in $G$. Let $x, y \notin S, x \neq y$. Then $x, y \in C$ and there exists $w \in V(G) \backslash C$ such that $x y \in E(G)$ and $w \in N_{G}(x) \backslash N_{G}(y)$ or $w \in N_{G}(y) \backslash N_{G}(x)$. By Remark 5, $x \in I_{K_{1}+G}[y, w]$ or $y \in I_{K_{1}+G}[x, w]$, showing that $S$ is a strong resolving dominating set of $K_{1}+G$.

Suppose $S=\left(V(G) \backslash C^{*}\right) \cup\left\{z \in C^{*} ; \operatorname{deg}_{G}(z)=n-1\right\}$, where $C^{*}$ is a dominated superclique in $G$. Let $x, y \notin S, x \neq y$. Then $x, y \in C^{*}$. By the same argument above, there exists $w \in S$ that strongly resolves $x$ and $y$. Now, consider the vertices $x$ and $v$. Since $x \notin S$, then $\operatorname{deg}_{G}(x)<n-1$. Hence, there exists $z \in V(G)$ such that $x z \notin E(G)$. It follows that $v \in I_{K_{1}+G}[x, z]$. Thus, $S$ is a strong resolving dominating set of $K_{1}+G$.

Corollary 1. Let $P_{n}=\left[v_{1}, v_{2}, \ldots, v_{n}\right]$ and $C_{m}=\left[c_{1}, c_{2}, \ldots, c_{m}, c_{1}\right]$ where $n, m \geq 3$.

(i) The sets $V\left(P_{n}\right) \backslash\left\{v_{i}, v_{i+1}\right\}$, for $i=2 \ldots, n-2$ are the strong resolving dominating sets of $\langle v\rangle+P_{n}$.

(ii) The sets $V\left(C_{m}\right) \backslash\left\{c_{i}, c_{i+1}\right\}$ and $V\left(C_{m}\right) \backslash\left\{c_{1}, c_{m}\right\}$, for $i=1,2 \ldots, m-1$ are the strong resolving dominating sets of $\langle v\rangle+C_{m}$.

Corollary 2. Let $G$ be a nontrivial connected graph of order $n$. Then

(i) for $\gamma(G)=1$, we have $\gamma_{s r}\left(K_{1}+G\right)=n-\omega_{S}(G)+1$;

(ii) for $\gamma(G) \neq 1$, we have $\gamma_{s r}\left(K_{1}+G\right)=\min \left\{\gamma_{s r}(G), n-\omega_{S}(G)\right\}$.

The next result follows from Proposition 3, Theorem 1 and Theorem 2.

Corollary 3. Let $G$ be nontrivial connected graph with $\operatorname{diam}(G) \leq 2$. Then

(i) for $\gamma(G)=1$, we have $\gamma_{s r}\left(K_{1}+G\right)=\operatorname{sdim}(G)+1$;

(ii) for $\gamma(G) \neq 1$, we have $\gamma_{s r}\left(K_{1}+G\right)=\min \left\{\gamma_{s r}(G), \operatorname{sdim}(G)+1\right\}$.

The following theorem gives a characterization of the strong resolving dominating sets in the join of $K_{1}$ and a disconnected graph $G$.

Theorem 3. Let $K_{1}=\langle v\rangle$ and $G$ be a disconnected graph whose components are $G_{i}$ for $i=1,2, \ldots, m$. A proper subset $S$ of $V\left(K_{1}+G\right)$ is a strong resolving dominating set of $K_{1}+G$ if and only if $S=V(G)$ or $S=V(G) \backslash C_{i}^{*}$ or $S=V\left(K_{1}+G\right) \backslash C_{i}$ where $C_{i}$ is a superclique in $G_{i}$, for $i=1,2, \ldots, m$ and $C_{i}^{*}$ is a dominated superclique of $G_{i}$.

Proof: Let $S$ be a strong resolving dominating set of $K_{1}+G$. Suppose $v \notin S$. Then $S \subsetneq$ $V(G)$. Let $C_{i}=V\left(K_{1}+G\right) \backslash S$, for $i=1,2, \ldots, m$. Then $S=V\left(K_{1}+G\right) \backslash C_{i}=V(G) \backslash C_{i}$. Let $x, y \in C_{i}, x \neq y$. Since $d_{K_{1}+G}(w, x)=d_{K_{1}+G}(w, y)$, for all $w \in V(G) \backslash V\left(G_{i}\right)$, there exists $z \in V\left(G_{i}\right) \backslash C_{i}$ such that $x \in I_{G_{i}}[y, z]$ or $y \in I_{G_{i}}[x, z]$. By Remark 5, 
$x \in N_{G_{i}}(y) \backslash N_{G_{i}}(z)$ or $y \in N_{G_{i}}(x) \backslash N_{G_{i}}(z)$. Thus, $C_{i}$ is a superclique in $G_{i}$. Since $\{v\}$ is a superclique in $K_{1}+G$, by Proposition $3, S=\left(V\left(K_{1}+G\right) \backslash\{v\}\right)=V(G)$. On the other hand, if $v \in S$ and $C_{i}=V\left(K_{1}+G\right) \backslash S$, for $i=1,2, \ldots, m$, then $S=V\left(K_{1}+G\right) \backslash C_{i}$, where $C_{i}$ is a superclique in $K_{1}+G$, by Proposition 3. Hence, $C_{i}$ is a superclique in $G_{i}$. Similarly, if $C_{i}^{*}=V(G) \backslash S$ for $i=1,2, \ldots, m$ where $C_{i}^{*}$ is a dominated superclique of $G_{i}$ and since $S$ is dominating, then $V\left(G_{i}\right) \backslash C_{i}^{*}$ is a dominating set of $G_{i}$.

For the converse, if $S=V(G)$, then we are done. Suppose $S=V(G) \backslash C_{i}^{*}$, or $S=$ $V\left(K_{1}+G\right) \backslash C_{i}$, where $C_{i}$ and $C_{i}^{*}$ are superclique and dominated superclique, respectively, in $G_{i}$ for $i=1,2, \ldots, m$. Then by Theorem 1 and Theorem $2, V\left(G_{i}\right) \backslash C_{i}^{*}$ is a strong resolving dominating set of $K_{1}+G_{i}$. By Remark 4, $S=V\left(K_{1}+G\right) \backslash C_{i}$ is a strong resolving dominating set of $K_{1}+G$.

Corollary 4. Let $G_{i}$ be connected graphs of orders $n_{i}$ and $G$ be a disconnected graph whose components are $G_{i}$ for $i=1,2, \ldots, m$ and $S_{i}=V\left(G_{i}\right) \backslash C_{i}$ where $C_{i}$ is a maximum dominated superclique of $G_{i}$. Then

$$
\gamma_{s r}\left(K_{1}+G\right)=\sum_{i=1}^{m} n_{i}-\max \left\{\gamma_{s r}\left(G_{i}\right), \omega_{D S}\left(G_{i}\right)+1 \mid i=1,2, \ldots, m\right\} .
$$

In the join of two graphs $G$ and $H$, the previous results have already considered the case when $G$ or $H$ is trivial. Hence, in the following theorem, a characterization of the strong resolving dominating sets in the join of nontrivial connected graphs $G$ and $H$ is considered.

Theorem 4. Let $G$ and $H$ be nontrivial connected graphs of orders $m$ and $n$, respectively. A proper subset $S$ of $V(G+H)$ is a strong resolving dominating set of $G+H$ if and only if at least one of the following is satisfied:

(i) $S=V(G+H) \backslash C_{G}$ where $C_{G}$ is a superclique in $G$.

(ii) $S=V(G+H) \backslash C_{H}$ where $C_{H}$ is a superclique in $H$.

(iii) If $\gamma(G)=1$ and $\gamma(H)=1$,

$$
\begin{aligned}
& S=\left[V(G+H) \backslash\left(C_{G} \cup C_{H}\right)\right] \cup\left\{z \in C_{G}: \operatorname{deg}_{G}(z)=m-1\right\}, \text { or } \\
& S=\left[V(G+H) \backslash\left(C_{G} \cup C_{H}\right)\right] \cup\left\{w \in C_{H}: \operatorname{deg}_{H}(w)=n-1\right\}
\end{aligned}
$$

where $C_{G}$ and $C_{H}$ are supercliques in $G$ and $H$, respectively.

(iv) If $\gamma(G) \neq 1$ and $\gamma(H) \neq 1$,

$$
S=\left[V(G+H) \backslash\left(C_{G} \cup C_{H}\right)\right]=\left(V(G) \backslash C_{G}\right) \cup\left(V(H) \backslash C_{H}\right),
$$

where $C_{G}$ and $C_{H}$ are supercliques in $G$ and $H$, respectively. 
Proof: Let $S$ be a strong resolving dominating set of $G+H$. Since $d_{G+H}(x, y)=1$, for each $x \in V(G)$ and $y \in V(H)$, none of the vertices in $V(G)$ and $V(H)$ strongly resolves any pair of distinct vertices in $V(H)$ and $V(G)$, respectively. Thus, $S \cap V(G) \neq \varnothing$ and $S \cap V(H) \neq \varnothing$. If $S \cap V(H)=V(H)$, then $S \neq V(G)$. Let $C_{G}=V(G) \backslash S$. Hence, $S=V(G+H) \backslash C_{G}$. Let $u, v \in C_{G}, u \neq v$. Then there exists $w \in S \cap V(G)$ such that $u \in I_{G+H}[v, w]$ or $v \in I_{G+H}[u, w]$. By Remark $5, w \in N_{G}(u) \backslash N_{G}(v)$ or $w \in N_{G}(v) \backslash N_{G}(u)$. Thus, $C_{G}$ is a superclique in $G$. Similarly, $S \cap V(G)=V(G)$. On the other hand, if $S \cap V(G) \neq V(G), S \cap V(H) \neq V(H), C_{G}=V(G) \backslash S$ and $C_{H}=V(H) \backslash S$, then $S=V(G+H) \backslash\left(C_{G} \cup C_{H}\right)$. Hence, $C_{G}$ and $C_{H}$ are supercliques in $G$ and $H$, respectively.

Suppose $\gamma(G)=1$ and $\gamma(H)=1$. Then

$$
A_{G}=\left\{z_{G} \in V(G) ; \operatorname{deg}_{G}(z)=m-1\right\} \neq \varnothing
$$

and

$$
A_{H}=\left\{z_{H} \in V(H) ; \operatorname{deg}_{H}(z)=m-1\right\} \neq \varnothing .
$$

By Proposition $2,\left|C_{G} \cap A_{G}\right| \leq 1$ and $\left|C_{H} \cap A_{H}\right| \leq 1$. Hence, we may assume that there exists $z_{G} \in C_{G} \cap A_{G}$ and $z_{H} \in C_{H} \cap A_{H}$. Then none of the vertices in $S \cap V(G)$ and $S \cap V(H)$ strongly resolves $z_{G}$ and $z_{H}$, a contradiction. Thus $z_{G} \in S$ or $z_{H} \in S$ so that $S=\left[V(G+H) \backslash\left(C_{G} \cup C_{H}\right)\right] \cup\left\{z \in C_{G}: \operatorname{deg}_{G}(z)=m-1\right\}$, or $S=\left[V(G+H) \backslash\left(C_{G} \cup\right.\right.$ $\left.\left.C_{H}\right)\right] \cup\left\{w \in C_{H}: \operatorname{deg}_{H}(w)=n-1\right\}$.

Suppose $\gamma(G) \neq 1$ or $\gamma(H)=1$. Then $A_{G}=\varnothing$ or $A_{H}=\varnothing$. Hence,

$$
S=V(G+H) \backslash\left(C_{G} \cup C_{H}\right)=\left(V(G) \backslash C_{G}\right) \cup\left(V(G) \backslash C_{G}\right) .
$$

Conversely, suppose $S$ satisfies condition $(i)$. Since $C_{G}$ is a superclique in $G$, there exists $w \in\left(V(G) \backslash C_{G}\right) \subseteq S$ such that $u \in I_{G}[v, w]$ or $v \in I_{G}[u, w]$, for any $u, v \notin S, u \neq v$, showing that $w$ strongly resolves $u, v$. A similar argument applies if $S$ satisfies condition (ii).

Suppose $S$ satisfies condition (iii) or (iv). Let $u, v \notin S, u \neq v$. If $u, v \in C_{G}$ or $u, v \in C_{H}$, then we are done. Consider the pair $u \in V(G) \backslash S$ and $v \in V(H) \backslash S$. Since $C_{G}$ is a superclique in $G$, there exists $z \in\left(V(G) \backslash C_{G}\right) \subseteq S$ such that $z \in N_{G}(u) \backslash N_{G}(v)$ or $z \in N_{G}(v) \backslash N_{G}(u)$. Hence, $u \in I_{G}[v, z]$ or $v \in I_{G}[u, z]$. Thus, $S$ is a strong resolving dominating set of $G+H$.

Corollary 5. Let $G$ and $H$ be nontrivial connected graphs of orders $m$ and $n$, respectively. Then

$$
\gamma_{s r}(G+H)= \begin{cases}\left(m-\omega_{S}(G)\right)+\left(n-\omega_{S}(H)\right)+1, & \text { if } \gamma(G)=1 \text { and } \gamma(H)=1 \\ \left(m-\omega_{S}(G)\right)+\left(n-\omega_{S}(H)\right), & \text { if } \gamma(G) \neq 1 \text { or } \gamma(H) \neq 1\end{cases}
$$

Remark 7. If $G$ is a nontrivial connected graph with $\gamma(G)=1$, then $\operatorname{diam}(G) \leq 2$.

Corollary 6. Let $G$ and $H$ be nontrivial connected graphs with $\gamma(G)=1$ and $\gamma(H)=1$. Then $\gamma_{s r}(G+H)=\operatorname{sdim}(G)+\operatorname{sdim}(H)+1$. In particular, 
(i) $\gamma_{s r}(G+H)=3$ for $G=P_{m}$ and $H=P_{n} \quad(m \geq 2, n \geq 2)$;

(ii) $\gamma_{s r}(G+H)=\left\lceil\frac{n}{2}\right\rceil+2$ for $G=P_{m}$ and $H=C_{n} \quad(m \geq 2, n \geq 3)$;

(iii) $\gamma_{s r}(G+H)=4$ for $G=C_{m}$ and $H=C_{n} \quad(m=n=3)$

(iv) $\gamma_{s r}(G+H)=\left\lceil\frac{m}{2}\right\rceil+\left\lceil\frac{n}{2}\right\rceil+1$ for $G=C_{m}$ and $H=C_{n} \quad(m, n \geq 4)$

Theorem 5. Let $G$ be a disconnected graph with components $G_{1}, \ldots, G_{n}$ and $H$ a disconnected graph with components $H_{1}, \ldots, H_{m}$. A proper subset $S$ of $V(G+H)$ is a strong resolving dominating set of $G+H$ if and only if $S$ satisfies any of the following:

(i) $S=S_{G} \cup V(H)$ where $V(G) \backslash S_{G}$ is a superclique of $G_{i}$ for some $i \in\{1,2, \ldots, n\}$;

(ii) $S=S_{H} \cup V(G)$ where $V(H) \backslash S_{H}$ is a superclique of $H_{j}$ for some $j \in\{1,2, \ldots, m\}$;

(iii) $S=S_{G} \cup S_{H}$, where $V(G) \backslash S_{G}$ and $V(H) \backslash S_{H}$ are supercliques of $G_{i}$ and $H_{j}$, for some $i \in\{1,2, \ldots, n\}$ and some $j \in\{1,2, \ldots, m\}$.

Proof: Let $S$ be a strong resolving dominating set of $G+H$ and $x \in G_{i}$ and $y \in G_{k}, i \neq$ $k$. Since $d_{G+H}(x, y)=2$ and $d_{G+H}(x, h)=d_{G+H}(y, h)=1$, for all $h \in V(H)$, then $x \in S$ or $y \in S$. Hence, $S \cap V(G)=\varnothing$. Similarly, $S \cap V(H) \neq \varnothing$. Let $S_{G}=S \cap V(G)$ and $S_{H}=S \cap V(H)$. Suppose $S \cap V(H)=V(H)$. Then $S_{G} \subseteq V(G)$. Let $C_{G}=V(G) \backslash S_{G}$. Then $S=S_{G} \cup V(H)$. Let $u, v \notin S_{G}, u \neq v$. Hence, $u, v \in C_{G}$. Since $S$ is a strong resolving dominating set of $G+H, C_{G} \subseteq V\left(G_{i}\right)$ for some $i \in\{1,2, \ldots, n\}$. Then there exists $z \in S \cap V\left(G_{i}\right)$ such that $u \in I_{G+H}[v, z]$ or $v \in I_{G+H}[u, z]$. It follows from Remark 5 that $C_{G}$ is a superclique of $G_{i}$. Similarly, $S \cap V(G)=V(G)$. On the other hand, if $S \cap V(G) \neq V(G), S \cap V(H) \neq V(H), C_{G}=V(G) \backslash S_{G}$ and $C_{H}=V(H) \backslash S_{H}$, then $S=S_{G} \cup S_{H}$. Hence, $C_{G}$ and $C_{H}$ are supercliques of $G_{i}$ and $H_{j}$ for some $i \in\{1,2, \ldots, n\}$ and $j \in\{1,2, \ldots, m\}$.

Conversely, suppose $S$ satisfies condition $(i)$. Let $u, v \notin S, u \neq v$. Then $u, v \in C_{G}=$ $V(G) \backslash S_{G}$. Hence, there exists $w \in V\left(G_{i}\right) \backslash C_{G}$ such that $w \in N_{G+H}(u) \backslash N_{G+H}(v)$ or $w \in N_{G+H}(v) \backslash N_{G+H}(u)$. By Remark $5, u \in I_{G+H}[v, w]$ or $v \in I_{G+H}[u, w]$. Thus, $S$ is a strong resolving dominating set of $G+H$. Similarly, the same conclusion holds if $S$ satisfies condition (ii).

Suppose $S$ satisfies condition (iii). Let $u, v \notin S, u \neq v$. If $u, v \in C_{G}$ or $u, v \in C_{H}$, then we are done. Assume $u \in C_{G}$ and $v \in C_{H}$. Since $d_{G+H}\left(u, u^{\prime}\right)=2$ for $u^{\prime} \in G_{k}, k \neq i$ and $d_{G+H}\left(v, v^{\prime}\right)=2$ for $v^{\prime} \in H_{p}, p \neq j, v \in I_{G+H}\left[u, u^{\prime}\right]$ or $u \in I_{G+H}\left[v, v^{\prime}\right]$. Thus, $S$ is a strong resolving dominating set of $G+H$.

\section{On Strong Resolving Domination in the Corona of Graphs}

The corona of two graphs $G$ and $H$, denoted by $G \circ H$, is the graph obtained by taking one copy of $G$ of order $n$ and $n$ copies of $H$, and then joining every vertex of the $i$ th copy of $H$ to the $i$ th vertex of $G$. For $v \in V(G)$, denote by $H^{v}$ the copy of $H$ whose vertices are attached one by one to the vertex $v$. Subsequently, denote by $v+H^{v}$ the subgraph of the corona $G \circ H$ corresponding to the join $\langle\{v\}\rangle+H^{v}, v \in V(G)$. 
Remark 8. For the coronas $P_{n} \circ K_{1}$ and $C_{n} \circ K_{1}$, it can be verified easily that

$$
\gamma_{s r}\left(P_{n} \circ K_{1}\right)=\gamma_{s r}\left(C_{n} \circ K_{1}\right)=n, \forall n \geq 3 .
$$

Theorem 6. Let $G$ be a nontrivial connected graph and $H$ a connected graph. A proper subset $S$ of $V(G \circ H)$ is a strong resolving dominating set of $G \circ H$ if and only if one of the following holds:

(i) $S=A \cup\left(\cup_{u \in V(G)} V\left(H^{u}\right)\right)$ where $A \subseteq V(G)$;

(ii) $S=A \cup\left(\cup_{u \in V(G) \backslash\{v\}} V\left(H^{u}\right)\right) \cup B_{v}$ for a unique $v \in V(G)$, where $A \subseteq V(G) \backslash\{v\}$ and $B_{v}$ is a strong resolving dominating set of $H^{v}$ if $\gamma(H)=1$ or $B_{v}$ is a strong resolving dominating set of $\langle v\rangle+H^{v}$ if $\gamma(H) \neq 1$;

(iii) $S=A \cup\left(\cup_{u \in V(G) \backslash\{v\}} V\left(H^{u}\right)\right) \cup B_{v}$ for a unique $v \in V(G)$ where $v \in A \subseteq V(G)$ and $B_{v}$ is a strong resolving set of $H^{v}$ if $\gamma(H)=1$ and $B_{v}$ is a strong resolving set of $\langle v\rangle+H^{v}$ if $\gamma(H) \neq 1$.

Proof: Suppose $S$ is a strong resolving dominating set of $G \circ H$. Let $A=S \cap V(G)$ and $B_{v}=S \cap V\left(H^{v}\right)$, where $v \in V(G)$. Consider the following cases:

Case 1. $S \cap V\left(H^{v}\right)=V\left(H^{v}\right)$

Then $B_{v}=H^{v}$. Thus, $S=A \cup\left(\cup_{u \in V(G)} V\left(H^{u}\right)\right)$.

Case 2. $S \cap V\left(H^{v}\right) \neq V\left(H^{v}\right)$

Let $u, v \in V(G), u \neq v$ such that $S \cap V\left(H^{u}\right) \neq V\left(H^{u}\right)$ and $S \cap V\left(H^{v}\right) \neq V\left(H^{v}\right)$. Pick $p_{u} \in V\left(H^{u}\right) \backslash S$ and $p_{v} \in V\left(H^{v}\right) \backslash S$. Then, none of the vertices in $S$ strongly resolves $p_{u}$ and $p_{v}$, a contradiction. Thus, the vertex $v \in V(G)$ such that $S \cap V\left(H^{v}\right) \neq V\left(H^{v}\right)$ must be unique. Hence, $S=A \cup\left(\cup_{u \in V(G) \backslash\{v\}} V\left(H^{u}\right)\right) \cup B_{v}$.

Subcase $2.1 v \in S$

Let $C_{v}=V\left(H^{v}\right) \backslash B_{v}$. Hence, $B_{v}=V\left(H^{v}\right) \backslash C_{v}$. Then it can be verified that $C_{v}$ is a superclique in $H^{v}$. If $\gamma(H) \neq 1$, by Theorem $1, B_{v}$ is a strong resolving set of $\{v\}+H^{v}$. If $\gamma(H)=1$, then by Remark 7 and Theorem $2, B_{v}$ is a strong resolving set of $H^{v}$.

Subcase $2.2 v \notin S$

Since $S$ is a dominating set of $G \circ H, B_{v}$ is a dominating set of $H^{v}$. By similar argument in the proof of subcase 2.1, $B_{v}$ is a strong resolving dominating set of $H^{v}$ if $\gamma(H)=1$ or $B_{v}$ is a strong resolving dominating set of $\langle v\rangle+H^{v}$ if $\gamma(H) \neq 1$.

Conversely, suppose $(i),(i i)$ and (iii) hold. Consider the following cases:

Case 1. $p, q \in V(G) \backslash A$

Let $p, q \in V(G \circ H) \backslash S$ where $p \neq q$ and $p=v$ or $q=v$, but not both, then $p \in I_{G \circ H}[q, z]$ or $q \in I_{G \circ H}[p, z]$ for some $z \in B_{v}$. On the other hand, if $p \neq v$ and $q \neq v$, then $q \in I_{G \circ H}[p, w]$ for some $w \in V\left(H^{q}\right) \subset S$ or $p \in I_{G \circ H}[q, r]$ for some $r \in V\left(H^{p}\right) \backslash B_{v}$.

Case 2. $p, q \in V\left(H^{v}\right) \backslash B_{v}$

Since $B_{v}$ is a strong resolving set of $H^{v}$, there exists $t \in B_{v} \subset S$ that strongly resolves $p$ and $q$.

Case 3. $p \in V(G) \backslash(A \cup\{v\})$ and $q \in V\left(H^{v}\right) \backslash B_{v}$

Since $p \neq v$, then $V\left(H^{p}\right) \subset S$ and $p \in I_{G \circ H}[q, z]$ for all $z \in V\left(H^{p}\right)$. 
Case 4. $p=v, q \in V\left(H^{v}\right) \backslash B_{v}$

Let $t \in N_{G}(v)$. Then $V\left(H^{t}\right) \subset S$ and $p \in I_{G \circ H}[q, u]$, for some $u \in V\left(H^{t}\right)$.

Cases 1 to 4 imply that $S$ is a strong resolving dominating set of $G \circ H$ and (i), (ii), (iii) imply that $S$ is a dominating set of $G \circ H$. Accordingly, $S$ is a strong resolving dominating set of $G \circ H$.

Corollary 7. Let $G$ and $H$ be connected graphs of orders $m$ and $n$, respectively

$$
\gamma_{s r}(G \circ H)= \begin{cases}(m-1) n+\gamma_{s r}(H), & \text { if } \gamma(H)=1 \\ (m-1) n+\gamma_{s r}\left(K_{1}+H\right), & \text { if } \gamma(H) \neq 1\end{cases}
$$

\section{Acknowledgements}

This research is funded by the Commission on Higher Education (CHED) and Mindanao State University-Iligan Institute of Technology, Philippines.

\section{References}

[1] C. Berge. Theorie des graphes et ses applications. Metheun and Wiley, London and New York, 1962.

[2] E. Cockayne and S. Hedetniemi. Towards a theory of domination in graphs. Networks, $7(3): 247-261,1977$.

[3] A. Cuivillas and Jr. S. Canoy. Restrained double domination in the join and corona of graphs. International Journal of Math. Analysis, 8(27):1339-1347, 2014.

[4] F. Harary. Graph Theory. Addison-Wesley Publishing Company, USA, 1969.

[5] O. Oellermann and J. Peter-Fransen. The Strong Metric Dimension of Graphs and Digraphs. Discrete Applied Mathematics, 155(3):356-364, 2007.

[6] P. Slater. Dominating and reference sets in a graph. Journal of Mathematics and Physical Science, 22(4):445-455, 1988. 